\title{
Characterization of an Implicitly Resistively-Loaded Monopole Antenna in Lossy Liquid Media
}

\author{
Colleen J. Fox, Paul M. Meaney, Fridon Shubitidze, Lincoln Potwin, and Keith D. Paulsen \\ Thayer School of Engineering, Dartmouth College, Hanover, NH 03755, USA \\ Correspondence should be addressed to Paul M. Meaney, paul.meaney@dartmouth.edu
}

Received 7 September 2007; Accepted 18 January 2008

Recommended by Elise Fear

Microwave tomographic imaging of the breast for cancer detection is a topic of considerable interest because of the potential to exploit the apparent high-dielectric property contrast between normal and malignant tissue. An important component in the realization of an imaging system is the antenna array to be used for signal transmission/detection. The monopole antenna has proven to be useful in our implementation because it can be easily and accurately modeled and can be positioned in close proximity to the imaging target with high-element density when configured in an imaging array. Its frequency response is broadened considerably when radiating in the liquid medium that is used to couple the signals into the breast making it suitable for broadband spectral imaging. However, at higher frequencies, the beam patterns steer further away from the desired horizontal plane and can cause unwanted multipath contributions when located in close proximity to the liquid/air interface. In this paper, we have studied the behavior of these antennas and devised strategies for their effective use at higher frequencies, especially when positioned near the surface of the coupling fluid which is used. The results show that frequencies in excess of $2 \mathrm{GHz}$ can be used when the antenna centers are located as close as $2 \mathrm{~cm}$ from the liquid surface.

Copyright (c) 2008 Colleen J. Fox et al. This is an open access article distributed under the Creative Commons Attribution License, which permits unrestricted use, distribution, and reproduction in any medium, provided the original work is properly cited.

\section{INTRODUCTION}

Microwave imaging techniques have been investigated for biomedical applications for nearly three decades, but have received renewed interest because of their potential in breast imaging based on data which have shown significant property contrast between normal and malignant tissue [1-3]. Their exploration is particularly timely given the well documented limitations of X-ray mammography which is the standard of care for breast cancer screening in most developed countries [4]. While there have been several early reports on microwave radiometry as a detection scheme that exploits the elevated temperatures of tumors [5-7], more recent investigations have focused on tomographic and ultrawideband (UWB) radar approaches [8-17].

The UWB radar methods are intriguing because they may be able to capitalize on the considerable advances in microwave technology for UWB communications. Indeed, simulations have shown that detection of tumors as small as $2-3 \mathrm{~mm}$ in diameter may be possible $[10,18]$. In practice, implementation may prove to be more challenging because wideband antennas are typically large (i.e., spiral antennas) or resistively loaded in which case they often have very low efficiency that limits signal penetration depth for tumor detection [19-21], although novel antenna designs that are not resistively loaded but offer good efficiency over a narrower frequency band have been identified [19].

In terms of tomographic approaches, while there has been a considerable amount of numerical modeling $[9,14$, $16,17,22,23]$, only a few systems have been developed for phantom experiments [24-26] and for actual breast imaging $[13,15]$. Similarly to the radar methods, one of the important considerations is the antenna design. Some systems (e.g., Semenov et al. [26]) have utilized waveguide radiators positioned on a large diameter whereas others have implemented monopole antennas $[13,15]$. These antennas are submerged in lossy liquids (either saline or glycerin-water mixtures) and subsequently are resistively loaded and also have the associated efficiency degradation, although experience has shown that transmitted signals can still be detected down to roughly $-140 \mathrm{dBm}$ with well-designed, low-noise receivers [27].

The coaxial monopole antenna is attractive for microwave breast imaging because its size and shape allow it to be densely packed around an imaging target. The design, 
constructed by removing a length of the outer conductor at one end of a rigid coaxial cable, is simple and low cost. In general, the monopole has a relatively narrow bandwidth and excites surface currents along its outer conductor $[28,29]$ in air where it closely resembles a dipole when baluns and/or notched outer conductors are applied [30]. However, when placed in a lossy medium the behavior changes dramatically, and its bandwidth increases significantly with the associated resistive loading. Additionally, we have found that the rigid coaxial cable interfaces well with an imaging configuration where liquid coupling and vertical positioning are required.

The goal of this investigation is to assess monopole antenna performance. Of particular, concern has been the measurement behavior when the antennas approach the liquid surface. Images near the surface correspond to planes closest to the chest wall for breast exams and are important given the relatively high-cancer incidence in the axilla region which is adjacent to the chest wall. When the antennas are fully submerged in a homogeneous coupling bath, the measurement magnitudes generally decrease monotonically as a function of the distance between transmit and receive antennas [27]. However, this pattern degrades progressively as the antennas approach the liquid surface and is most pronounced for the higher end of our operating frequency range (generally above $1500 \mathrm{MHz}$ ). As a result, we have investigated the characteristics of these antennas with respect to the most readily available parameters-composition of the coupling bath and antenna length-in order to devise strategies for improving overall performance. In general, we have found strong correlations between the return loss, the effective beam width, and beam steering angle as a function of antenna electrical length which is consistent over multiple bath compositions (i.e., with significantly different permittivity and conductivity). In addition, these measurements are consistent with trends observed in associated simulation results. Based on these studies, we have optimized the antenna design to allow operation relatively close to the liquid surface over the upper end of our operating frequency range.

\section{METHODS}

Our breast imaging system operates in a range of liquid coupling solutions with varying electrical permittivities to improve the dielectric property match to specific breast density types. Previous results have demonstrated significant variations in permittivity and conductivity as a function of breast density [31] primarily due to the overall higher-water content of the fibroglandular tissue compared with fat [32-34]. Breast types vary over a continuum from fatty (mostly adipose tissue) to extremely dense (mostly fibroglandular tissue) [35]. In light of the variations in breast parenchymal density, we present data for multiple bath compositions in order to illustrate the consistency of our techniques. We refer the interested reader to previous publications for a detailed description of our clinical breast imaging system $[13,27]$.

\subsection{Data calibration}

As part of the calibration procedure for our breast imaging system, a set of measurement data is acquired for the ho-

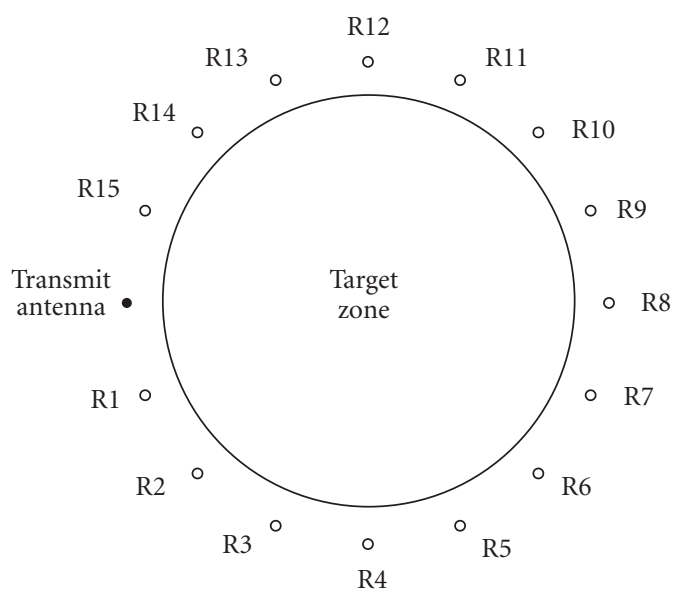

FIGURE 1: 2D schematic diagram of the 16 monopole antennas arranged in a $15.2 \mathrm{~cm}$ diameter circular array surrounding the imaging zone.

mogeneous bath for all operating frequencies and all possible permutations of transmit and receive antennas. Figure 1 shows the circular antenna array configuration used during breast imaging exams. Beyond minor interreceiver channel gain variations, the magnitudes exhibit a predictable behavior for a given transmitter where power levels decrease monotonically with distance. Figures 2(a) and 2(b) show the amplitude measurements with respect to receiver antenna number for a single transmitter over a range of frequencies for the cases of the array: (a) at its maximum depth below the 87 : 13 glycerin-water bath surface (position 7), and (b) with the antenna centers just $2 \mathrm{~cm}$ below the liquid surface (position 1). Note that all 16 antennas in the array are positioned on a $15.2 \mathrm{~cm}$ diameter circle with an orientation such that relative receivers 1 and 15 are adjacent to the transmitter while receiver 8 is furthest away. The plots for frequencies from 500 to $1700 \mathrm{MHz}$ in Figure 2(a) suggest a monotonic signal decrease with respect to transmitter/receiver separation distance. However, the corresponding curves in Figure 2(b) exhibit significant variations primarily at the higher frequencies and for the larger antenna separations. Given that the only difference between the two cases is the depth of the antennas below the liquid surface, the most likely explanation is that unwanted multipath signals (related to coupling at the bath/air interface) are causing the perturbations.

\subsection{Beam characterization}

The horizontal beam pattern of a monopole antenna is isotropic due to its radial symmetry; however, the vertical pattern in a lossy solution is considerably more complex. The goal of the first set of experiments was to characterize the vertical plane beam width and beam center with respect to wavelength. This was accomplished by transmitting from a single, vertically oriented, monopole antenna and recording the amplitudes measured by a receiving waveguide antenna at different vertical positions. A waveguide antenna was useful in this 

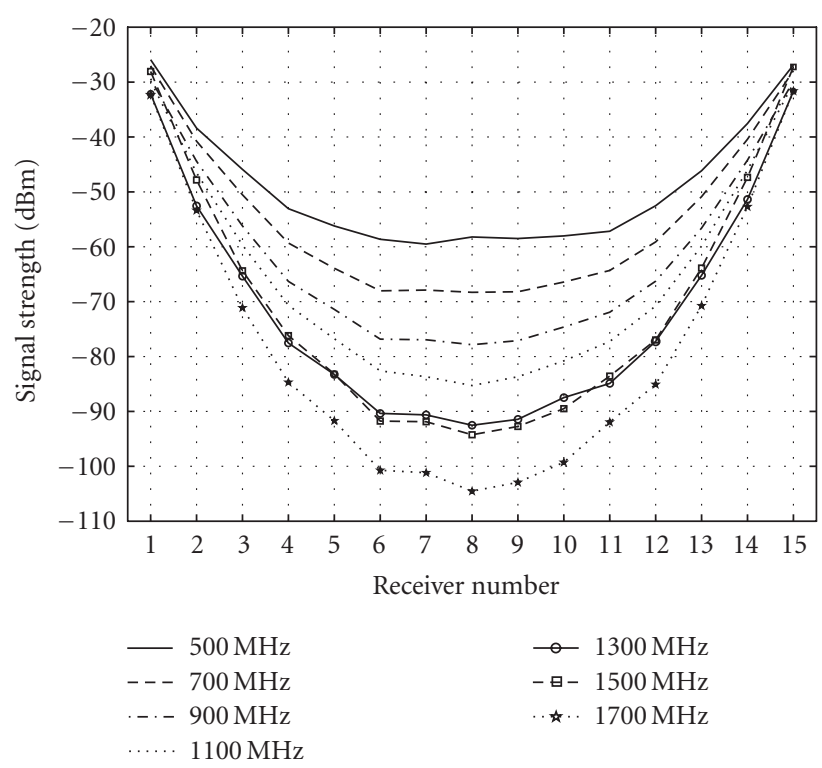

○ $1300 \mathrm{MHz}$
- $1500 \mathrm{MHz}$
. $1700 \mathrm{MHz}$

(a)

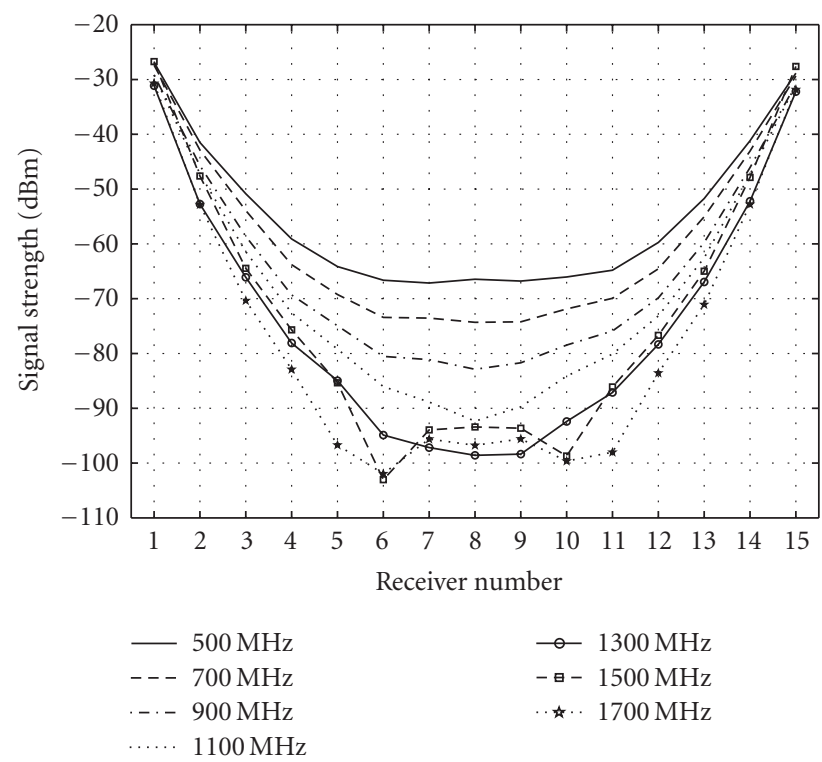

(b)

FIGURE 2: Signal amplitude versus relative receiver number associated with a single transmitting, $3.4 \mathrm{~cm}$ long antenna for a range of frequencies when the antenna center is submerged (a) $10 \mathrm{~cm}$ and (b) $2 \mathrm{~cm}$ below the $87 \%$ glycerin coupling liquid surface, respectively.

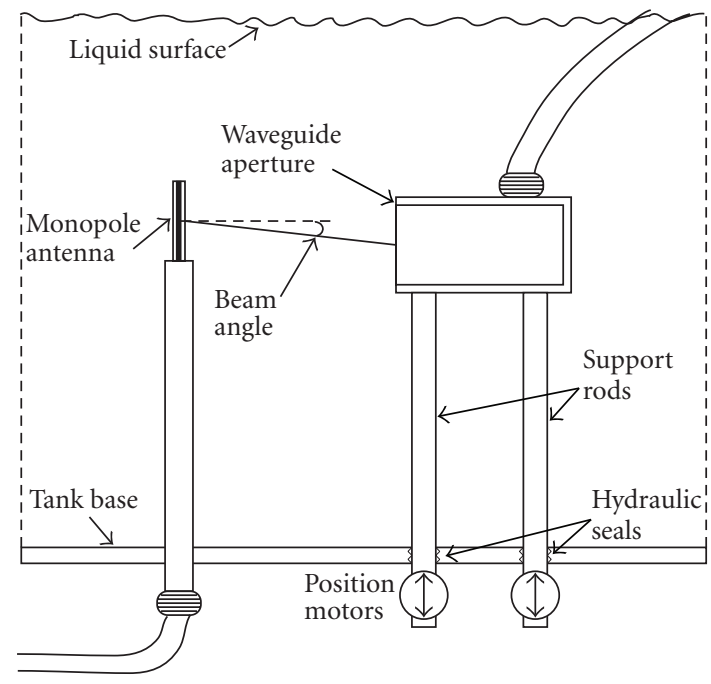

(a)

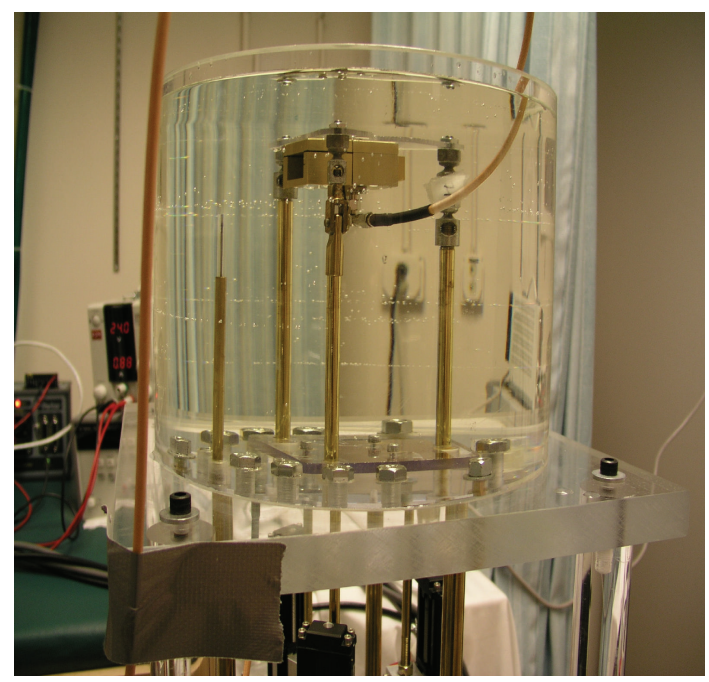

(b)

FIGURE 3: (a) Schematic diagram and (b) photograph of the setup for beam pattern measurement involving a 3-point support (3PS) mechanical steering system with the aperture reference waveguide antenna positioned approximately $6 \mathrm{~cm}$ from the monopole transmitter.

role because of its beam symmetry in the E-plane. The main variables in the investigation were the signal wavelength in the medium and the relative vertical position of the reference (waveguide) antenna. Secondary variables included the coupling medium and the length of the transmitting (monopole) antenna.

A 3-point support (3PS) mechanical steering system, developed by Meaney et al. [36], was used to position the reference antenna during these studies. Figures 3(a) and 3(b) show a schematic diagram and photograph of the 3PS system configured for the experiments. The waveguide was oriented perpendicularly to the axis of the monopole and was translated in vertical steps of $2.5 \mathrm{~mm}$ under computer control. We did not rotate the reference waveguide about the antenna under test as would be the case in more traditional beam characterization measurements because of the symmetry of the experiments and the logistical challenges of acquiring data in the lossy liquid bath.

The cylindrical testing tank was filled with a lossy glycerin and water solution similar to that used in clinical breast imaging. Measurements were taken in a $90 \%$ glycerin, $10 \%$ water solution and a $40 \%$ glycerin, $60 \%$ water solution to 


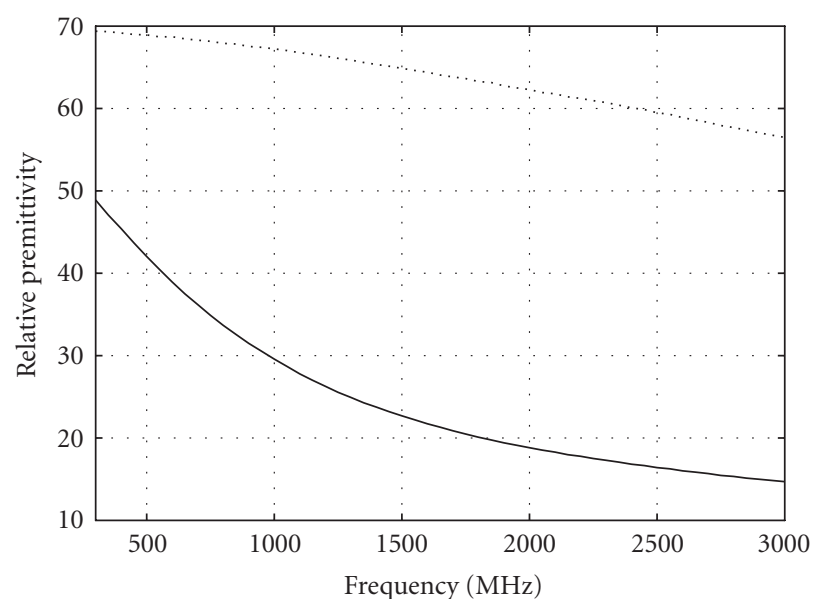

…. $40 \%$ glycerin - $90 \%$ glycerin

(a)

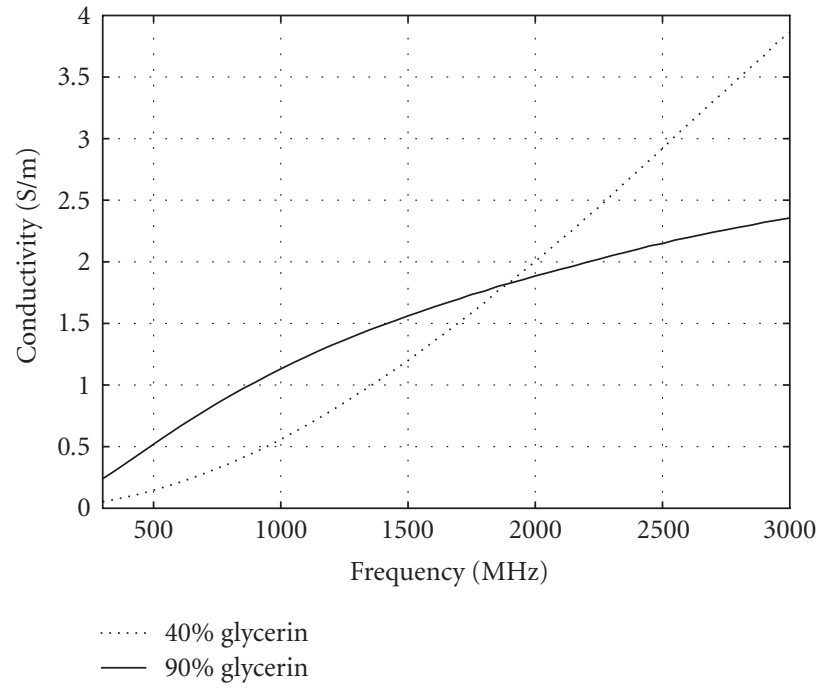

(b)

FIGURE 4: The measured (a) relative permittivity and (b) conductivity of the $90 \%$ and $40 \%$ glycerin: water baths used in these experiments.

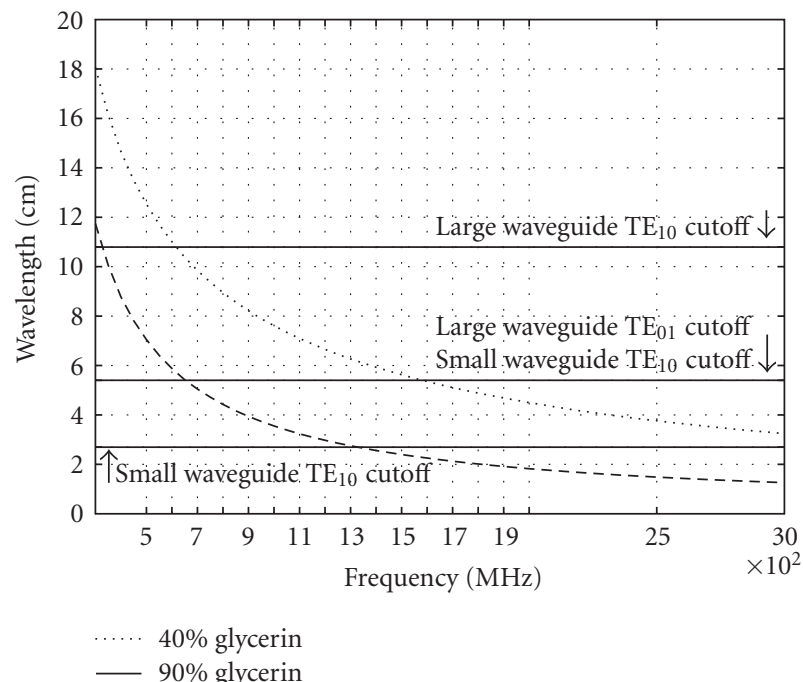

FIGURE 5: Plot of wavelength versus frequency for the $90 \%$ and $40 \%$ glycerin solutions along with the $\mathrm{TE}_{10}$ cutoff wavelengths for the receiving waveguides.

provide a range of complex permittivity values and resulting wavelengths similar to those observed in biological tissue. All measurements were collected using an Agilent 8753C network analyzer in sweep mode with a frequency range of $300 \mathrm{MHz}$ to $3 \mathrm{GHz}$.

Two complementary waveguide apertures were required to span the full frequency range of the monopole antennas under test. The operational wavelength cutoff for the waveguides was calculated using $1[29,37]$ :

$$
\lambda_{c}=\frac{2}{\sqrt{(m / a)^{2}+(n / b)^{2}}} \stackrel{\mathrm{TE}_{10}}{\longrightarrow} 2 a,
$$

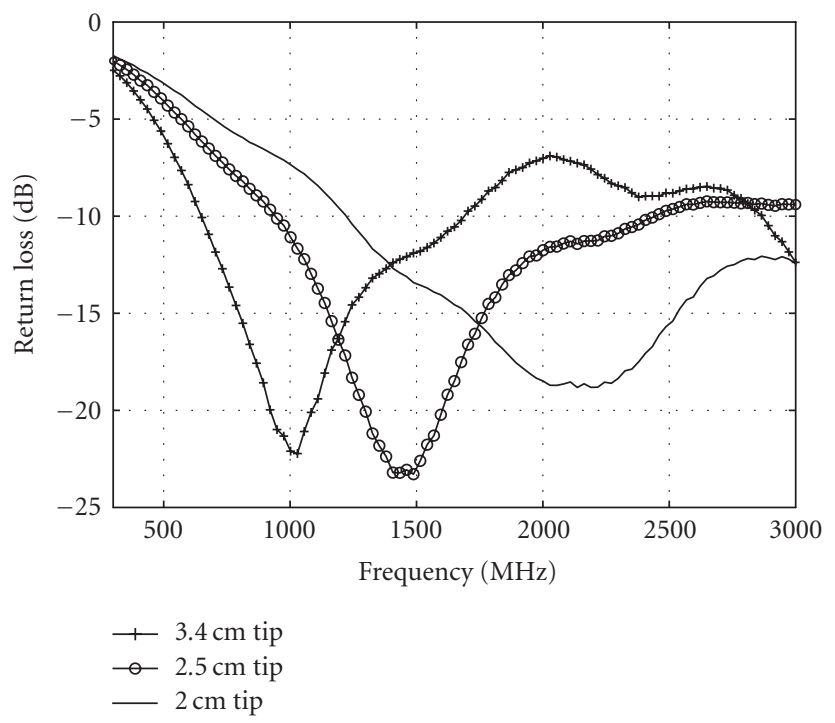

Figure 6: Measured return loss of the 2.0, 2.5, and $3.4 \mathrm{~cm}$ length monopole antennas in the $90 \%$ glycerin: water bath as a function of frequency.

where $m$ and $n$ are the mode indices, and $a$ and $b$ are the larger and smaller inner dimensions of the front face of the waveguide, respectively. The $\mathrm{TE}_{10}$ mode results in the lowest cutoff frequency and determines the lower operational limit of the waveguide. In this situation, the wavelength for a $\mathrm{TE}_{10}$ mode wave decreases to two times the width. Table 1 shows the dimensions of both receiving waveguide apertures along with their corresponding cutoff wavelengths. The dielectric properties for the $90 \%$ and $40 \%$ glycerin-water baths are shown in Figure 4. Given that these waveguide antennas were filled with the surrounding liquid, and that the complex permittivity of the glycerin-water solutions varied with frequency, it is instructive to plot the propagation wavelengths 


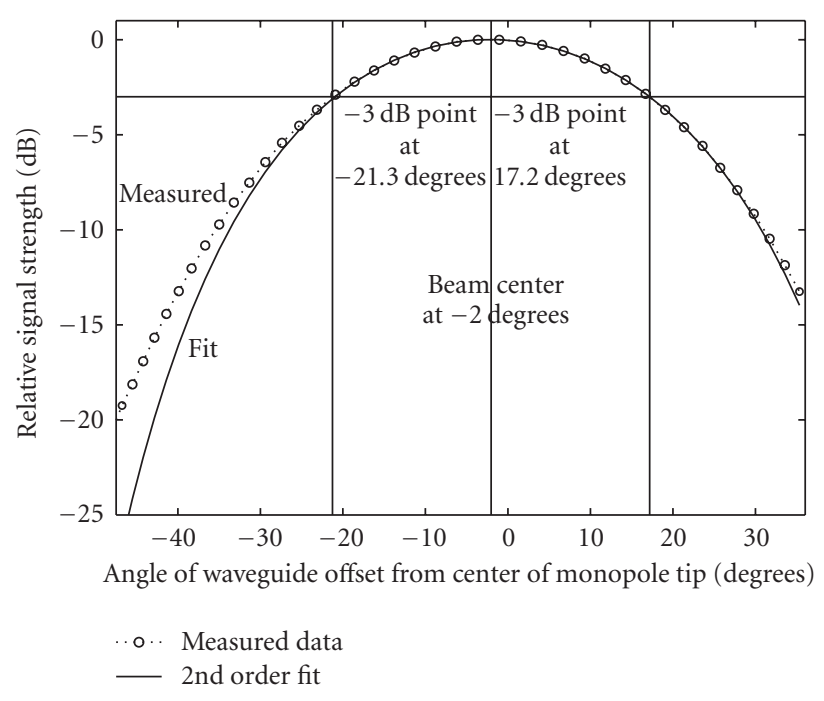

FIGURE 7: Vertical beam pattern for the $3.4 \mathrm{~cm}$ length monopole antenna at $1596 \mathrm{MHz}$ in a $90 \%$ glycerin: water bath. A 15-point 2nd order polynomial was used to estimate the beam center location and is also shown along with the points $3 \mathrm{~dB}$ below the peak value.

TABLE 1: Reference waveguide antenna dimensions and cutoff wavelengths.

\begin{tabular}{lccc}
\hline Waveguide & Width, $a(\mathrm{~cm})$ & Height, $b(\mathrm{~cm})$ & Cutoff $\lambda, 2 a(\mathrm{~cm})$ \\
\hline Large & 5.4 & 2.7 & 10.8 \\
Small & 2.7 & 1.4 & 5.4 \\
\hline
\end{tabular}

for both solutions versus frequency along with horizontal lines corresponding to the cutoff wavelengths of the waveguides (Figure 5). The points where the curves intersect indicate the $\mathrm{TE}_{10}$ and $\mathrm{TE}_{01}$ mode cutoff frequencies as the lower and upper operating frequency bounds, respectively. From this graph, it is evident that the large and small waveguides can be used from 610 to $1580 \mathrm{MHz}$ and from 1580 to greater than $3000 \mathrm{MHz}$, respectively, in the $90: 10$ glycerin-water bath. Likewise, for the $40: 60$ glycerin-water bath, the large and small waveguides can be used from 330 to $660 \mathrm{MHz}$ and from 660 to $1270 \mathrm{MHz}$, respectively.

\subsection{Antenna modeling}

In addition to the measurements used to characterize these antennas, we have also performed a series of numerical simulations that exhibited comparable behavior. For this modeling, we utilized a finite difference time domain (FDTD) approach in a cylindrical coordinate system which incorporated a rigid $50 \Omega$ coaxial feel line along with the geometry of the antenna radiating a lossy, semi-infinite medium [38]. The source was setup to excite only a transverse magnetic field in the coaxial line. The inner and outer conductors of the coaxial cable were represented through perfect electrical conducting (PEC) boundary conditions. The coaxial line dielectric was assumed to be lossless with a relative permittivity of 2.0, and the spatial resolution of the grid was estab- lished to represent accurately the vertical and radial components of the electric field within the coaxial cable. The dielectric properties of the surrounding bath were the same as those of the medium used in the measurements with the simulations being performed individually at each frequency due to the property dispersion over this range. Eight layers of a perfectly matching layer (PML) were used to minimize backscattered signals from the finite termination of the computational mesh [39]. The simulations were run until the solution had stabilized, after which a Fourier transform was performed at each of the measurement sites to recover the steady-state field values. Similarly to the actual measurements, the $z$-component of the electric field values was computed at a series of points along a vertical line parallel to the center of the monopole antenna in $3 \mathrm{~mm}$ increments.

\section{RESULTS AND DISCUSSION}

In this section, we first report on the general characteristics of the monopole antennas in terms of their return loss and beam characteristics (steering angle and beamwidth). We then illustrate deviations in the transmit data for antenna positions near the liquid surface for different tip lengths which demonstrate how the overall antenna performance can be improved.

\subsection{Return loss}

The basic monopole antenna consisted of a $50 \Omega$ rigid coaxial cable with the outer conductor stripped from the transmitting zone. Tip lengths of $2.0,2.5$, and $3.4 \mathrm{~cm}$ were tested with each having broad operating bandwidths associated with return losses nominally of less than $-10 \mathrm{~dB}$ across the band for both the $90 \%$ and $40 \%$ liquid coupling mixtures (plots of the return losses for the $90 \%$ glycerin bath are shown in Figure 6). As expected, the dominant null in the return loss associated with the resonant frequency shifts upwards as the length of the transmitting tip decreases $-3.4 \mathrm{~cm}$ length at $1 \mathrm{GHz}, 2.5 \mathrm{~cm}$ length at $1.45 \mathrm{GHz}$, and the $2.0 \mathrm{~cm}$ length at $2.15 \mathrm{GHz}$. The lower-frequency limit also shifts upwards with antenna length. At the higher end of this frequency span, the resistive loading of the antennas generally suppresses the return loss to $-10 \mathrm{~dB}$ or less.

\subsection{Beam characterization}

Figure 7 shows a plot of the beam pattern for the $3.4 \mathrm{~cm}$ length monopole antenna in the $90 \%$ bath at $1596 \mathrm{MHz}$ as a function of the angle between the monopole antenna and the reference waveguide antenna ( 0 degree was the horizontal plane cutting through the center of the monopole antenna) with the $3 \mathrm{~dB}$ points indicated on either side of the beam peak. A second-order polynomial fit to the beam pattern was superimposed to estimate the beam center locationonly the 15 points near the beam center were used in the 


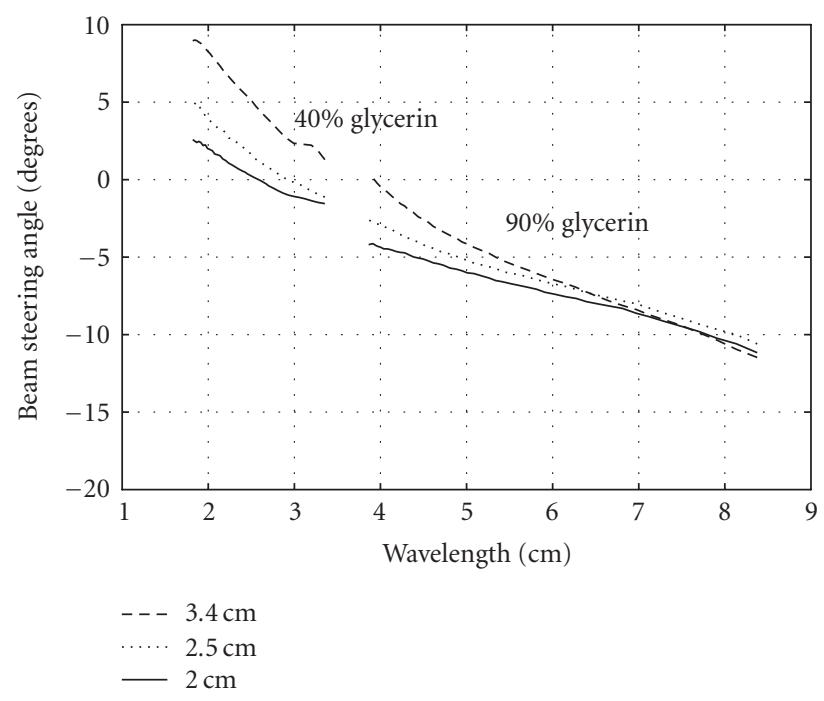

(a) Beam steering angles for the 2.0, 2.5, and $3.4 \mathrm{~cm}$ length monopole antennas as a function of the medium wavelength for the $90 \%$ and $40 \%$ glycerin: water baths

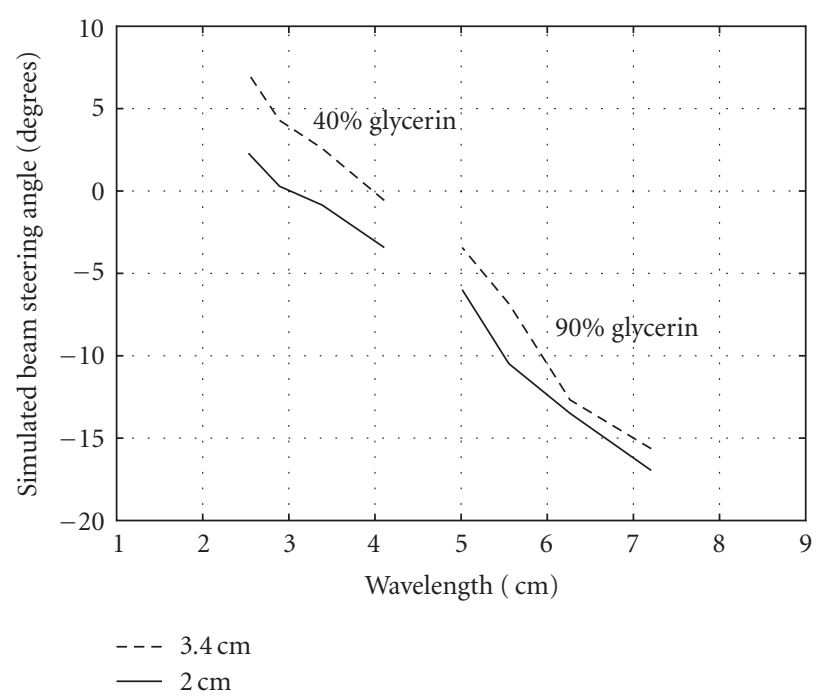

(b) Same as Figure 8(a) for simulation results of beam steering angles for the 2.0 and $3.4 \mathrm{~cm}$ length monopole antennas operating in the $90 \%$ and $40 \%$ glycerin: water baths

Figure 8

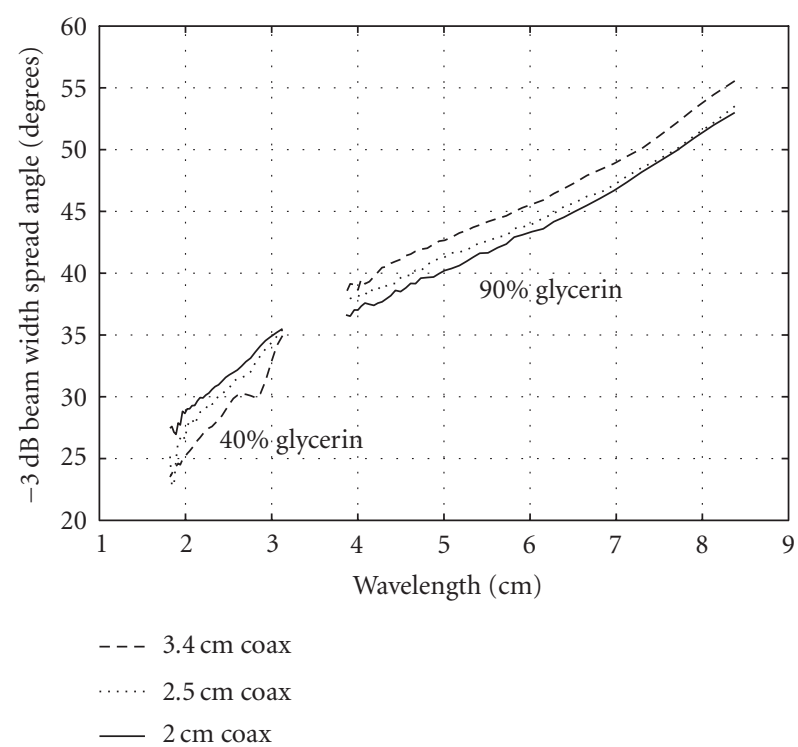

FIgURE 9: Beam widths for the 2.0, 2.5, and $3.4 \mathrm{~cm}$ length monopole antennas as a function of the medium wavelength for the $90 \%$ and $40 \%$ glycerin: water baths.

least squares fitting process. The beam center was readily extracted from the equation of the fitted curve. In terms of the $3 \mathrm{~dB}$ beam width, measurement points were selected which straddled the $3 \mathrm{~dB}$-power level below the peak in the beam pattern. From these two points, an interpolation was used to determine the closest position that would be exactly $3 \mathrm{~dB}$ below the peak.

Figure 8(a) shows plots of the beam center angle as a function of wavelength in the medium for the three antenna lengths in the two glycerin-water baths (90\% and $40 \%)$. (The vertical positions of the beam centers have been converted to beam angles.) The trends in these cases are clear. The beam steers increasingly upwards with decreasing operating wavelength (or increasing frequency). From these curves, it is evident that the beam steering is a function of antenna electrical length in the medium-longer lengths correspond to higher beam steering angles. These trends are echoed in the simulation results for the longest and shortest antenna lengths in the two electrically distinct baths shown in Figure 8(b). The computed behavior matches the measurement observations best at the higher frequencies of concern (i.e., where the beam steers upwards) and supports the validity of the measured behavior. Although the overall response with respect to the antenna lengths and bath properties is consistent between the experimental and simulated data at the longer wavelengths (lower frequencies), the simulated results suggest a more rapid downward steering effect as the wavelength lengthens than was found to occur with the actual measurement data.

Figure 9 shows the corresponding $3 \mathrm{~dB}$ beamwidths for the three different length monopole antennas in both the $90 \%$ and $40 \%$ glycerin-water baths as a function of medium wavelength. In general, the beamwidths increase significantly with wavelength and with different coupling baths (which also directly affects the bath wavelength). To a lesser degree, the beamwidth also appears to be a function of the antenna length. For the $90 \%$ bath, the beamwidth increase from the 2.0 to $3.4 \mathrm{~cm}$ length antenna is 2.5 degrees across the range of medium wavelengths. Interestingly, the effect for the $40 \%$ glycerin-water bath appears to be reversed with the beamwidths for the $2.0 \mathrm{~cm}$ monopole being broader than its $3.4 \mathrm{~cm}$ length counterpart. Given that the baths we typically use for breast imaging range from $79 \%$ to $90 \%$, the results from the $90 \%$ bath are more relevant. While beams steer higher with increasing frequency (potentially exacerbating the problem of the fields coupling to the liquid-air 


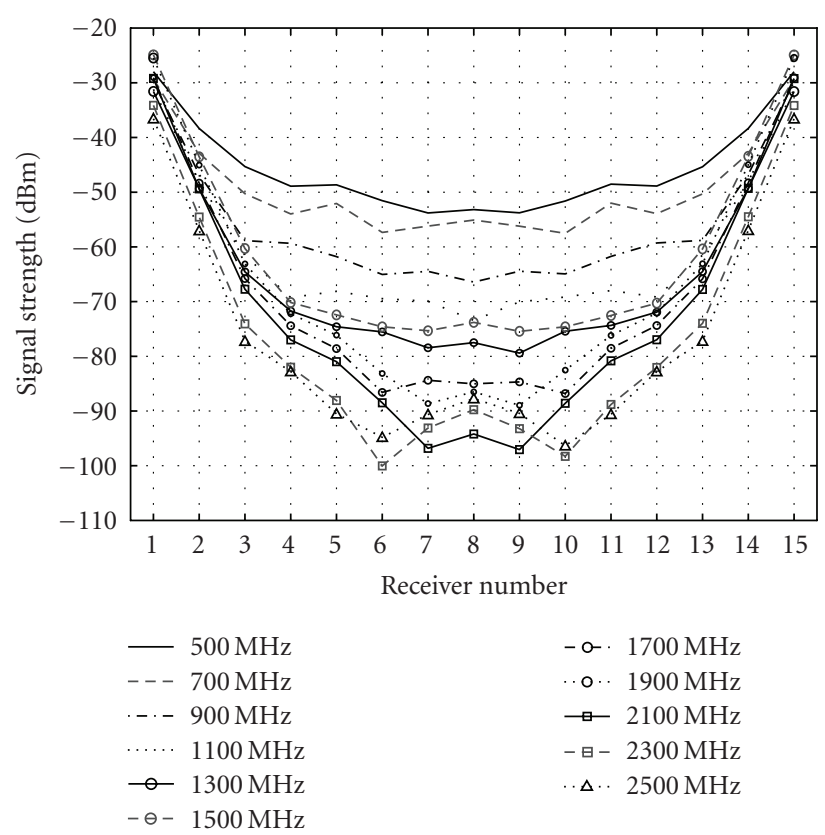

(a)

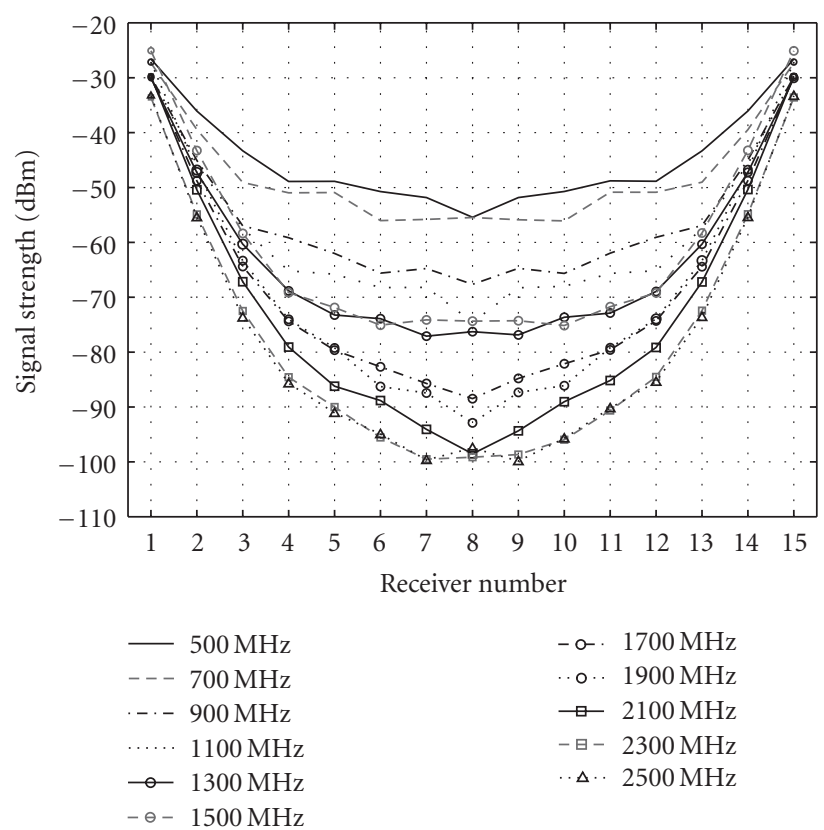

(b)

FIGURE 10: Plots of the transmitted signal strength as a function of receiver number over the frequency range of $500-2500 \mathrm{MHz}$ for the $3.4 \mathrm{~cm}$ length antennas with their centers submerged (a) $2.0 \mathrm{~cm}$ and (b) $6.5 \mathrm{~cm}$ below the $90 \%$ glycerin coupling liquid surface, respectively.

interface), the beamwidth narrows some with a shorter antenna length which counteracts the coupling effects at the liquid interface. Thus, it appears that a reduced antenna length causes the beam to steer lower while also slightly reducing the beamwidth.

\subsection{Multifrequency transmission characteristics}

Figure 10 shows transmission data from a single $3.4 \mathrm{~cm}$ length monopole to the corresponding receiver monopole antennas in the $90 \%$ glycerin bath for frequencies ranging from 500 to $2500 \mathrm{MHz}$ at antenna center depths of (a) 2.0 and (b) $6.5 \mathrm{~cm}$ below the liquid surface, respectively. For the deeper position, the shapes of the curves are nearly parabolic with the greater transmission loss corresponding to the greater propagation lengths (especially receiver antennas 7,8 , and 9). (Note that the array has 16 antennas evenly spaced in a circular arrangement). However, in the shallower case, the near-parabolic patterns are noticeably perturbed for the furthest receivers at frequencies above $1900 \mathrm{MHz}$.These aberrations are due to signals coupling to the liquid surface and propagating along an undesired, low loss surface path.

Figures 11(a) and 11(b) show the corresponding plots for the $2.0 \mathrm{~cm}$ length antennas. In this situation there appears to be a minor perturbation in the $2300 \mathrm{MHz}$ case and a more pronounced effect at $2500 \mathrm{MHz}$. These results are important because they suggest that we can operate at higher frequencies with reduced surface coupling effects using the shorter antennas. They also confirm the beam behavior exhibited in the previous section where the shorter length an- tennas steered towards lower positions minimizing the surface coupling. In addition, we plotted the average absolutevalued differences in the signal strength at the two antenna depths for the three furthest receivers as a function of frequency for the 3.4 and $2.0 \mathrm{~cm}$ antenna lengths (Figure 12). The deviation for the longer antenna appears to increase noticeably (i.e., nominally above $2 \mathrm{~dB}$ ) above $1900 \mathrm{MHz}$ while that for the $2.0 \mathrm{~cm}$ antenna only increases significantly at $2500 \mathrm{MHz}$.

\section{CONCLUSIONS}

We have assessed the antenna design to extend the usable operating frequency range of our clinical microwave breast imaging system. The primary difficulties have occurred in the imaging planes closest to the liquid surface where we have observed significant perturbations in the transmitted signals in the homogeneous case (which serves as the calibration data [27]). The planes near the liquid surface correspond to those closest to the chest wall in clinical breast exams which are important because of the high prevalence of cancer in the axilla zone. We have performed several experiments to determine the parameters of the monopole antennas which govern their overall performance. We have been able to alter the beam steering angle and beam width by modifying the antenna length while still maintaining our nominal operating frequency bandwidth. 


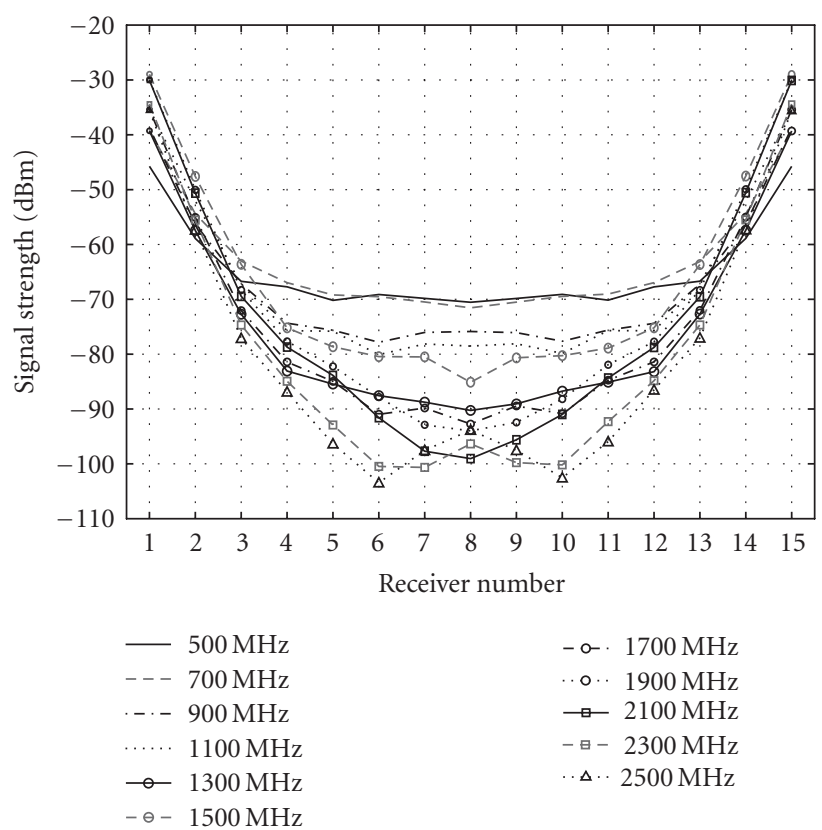

(a)

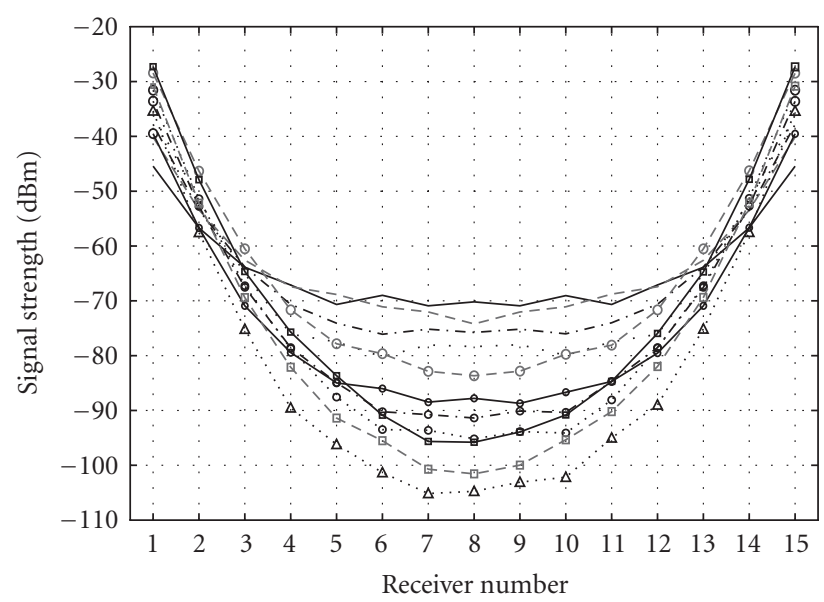

$-500 \mathrm{MHz}$
$---700 \mathrm{MHz}$
.$--900 \mathrm{MHz}$
$\ldots . .1100 \mathrm{MHz}$
$-\odot-1300 \mathrm{MHz}$
$-\ominus-1500 \mathrm{MHz}$

$-\circ-1700 \mathrm{MHz}$

. o.. $1900 \mathrm{MHz}$

$\rightarrow 2100 \mathrm{MHz}$

$-в-2300 \mathrm{MHz}$

. $\Delta .2500 \mathrm{MHz}$

(b)

FIGURE 11: Plots of the transmitted signal strength as a function of receiver number over the frequency range of $500-2500 \mathrm{MHz}$ for the $2.0 \mathrm{~cm}$ length antennas with their centers submerged (a) $2.0 \mathrm{~cm}$ and (b) $6.5 \mathrm{~cm}$ below the $90 \%$ glycerin coupling liquid surface, respectively.

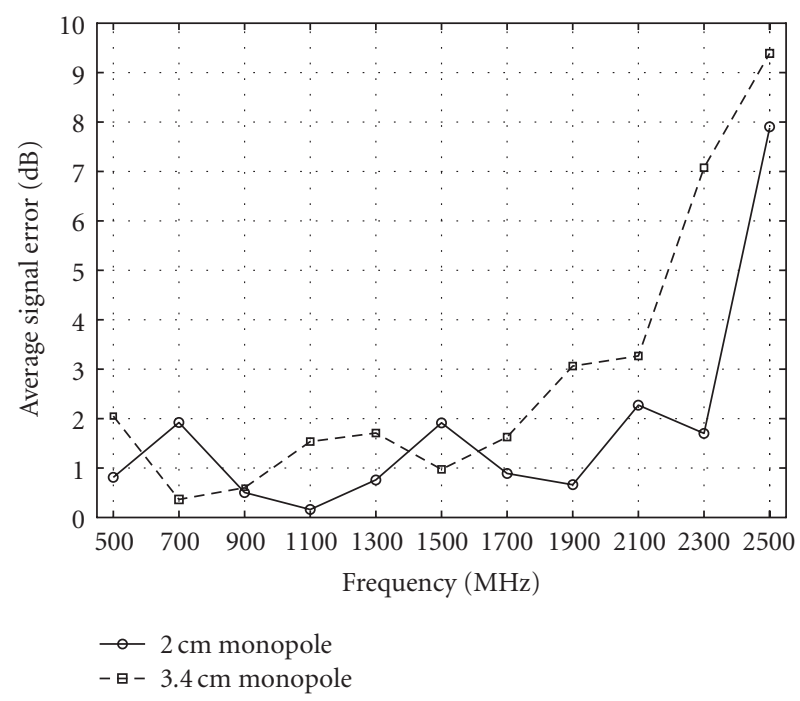

FIGURE 12: Plots of the average differences in signal strengths (utilizing only values for receivers 7,8 , and 9) between the shallow $(2.0 \mathrm{~cm})$ and deep $(6.5 \mathrm{~cm})$ measurement cases as a function of frequency for the 3.4 and $2.0 \mathrm{~cm}$ antenna lengths in the $90 \%$ glycerin coupling liquid.

The results comparing the transmission plots for the longer and shorter antennas clearly show that the shorter antennas can operate at higher frequencies for a given imaging plane close to the liquid surface. These improvements were achieved with minimal reduction in overall signal strength (less than $3 \mathrm{~dB}$ difference at the higher frequencies).

\section{ACKNOWLEDGMENT}

This work was supported by NIH/NCI Grant no. PO1CA80139.

\section{REFERENCES}

[1] S. S. Chaudhary, R. K. Mishra, A. Swarup, and J. M. Thomas, "Dielectric properties of normal and malignant human breast tissues at radiowave and microwave frequencies," Indian Journal of Biochemistry and Biophysics, vol. 21, no. 1, pp. 76-79, 1984.

[2] W. T. Joines, Y. Zhang, C. Li, and R. L. Jirtle, "The measured electrical properties of normal and malignant human tissues from 50 to $900 \mathrm{MHz}$," Medical Physics, vol. 21, no. 4, pp. 547550, 1994.

[3] A. J. Surowiec, S. S. Stuchly, J. R. Barr, and A. Swarup, "Dielectric properties of breast carcinoma and the surrounding tissues," IEEE Transactions on Biomedical Engineering, vol. 35, no. 4, pp. 257-263, 1988.

[4] J. E. Joy, E. E. Penhoet, and D. B. Petitti, Saving Women's Lives: Strategies for Improving Breast Cancer Detection and Diagnosis, The National Academy Press, Washington, DC, USA, 2005.

[5] P. C. Myers, A. H. Barrett, and N. L. Sadowsky, "Microwave thermography of normal and cancerous breast tissue," Annals of the New York Academy of Sciences, vol. 335, pp. 443-455, 1980.

[6] Y. Leroy, A. Mamouni, J. C. Van de Velde, B. Bocquet, and B. Dujardin, "Microwave radiometry for non-invasive thermometry," Automedica, vol. 8, pp. 181-202, 1987.

[7] K. L. Carr, P. Cevasco, P. Dunlea, and J. Shaeffer, "Radiometric sensing: an adjuvant to mammography to determine 
breast biopsy," in Proceedings of IEEE MTT-S International Microwave Symposium Digest, vol. 2, pp. 929-932, Boston, Mass, USA, June 2000.

[8] S. K. Davis, H. Tandradinata, S. C. Hagness, and B. D. Van Veen, "Ultrawideband microwave breast cancer detection: a detection-theoretic approach using the generalized likelihood ratio test," IEEE Transactions on Biomedical Engineering, vol. 52, no. 7, pp. 1237-1250, 2005.

[9] S. Caorsi, A. Massa, and M. Pastorino, "A computational technique based on a real-coded genetic algorithm for microwave imaging purposes," IEEE Transactions on Geoscience and Remote Sensing, vol. 38, no. 4, pp. 1697-1708, 2000.

[10] E. C. Fear and M. A. Stuchly, "Microwave detection of breast cancer," IEEE Transactions on Microwave Theory and Techniques, vol. 48, no. 11, pp. 1854-1863, 2000.

[11] R. Benjamin, I. J. Craddock, G. S. Hilton, et al., "Microwave detection of buried mines using non-contact, synthetic nearfield focusing," IEE Proceedings: Radar, Sonar and Navigation, vol. 148, no. 4, pp. 233-240, 2001.

[12] S. P. Poplack, T. D. Tosteson, W. A. Wells, et al., "Electromagnetic breast imaging: results of a pilot study in women with abnormal mammograms," Radiology, vol. 243, no. 2, pp. 350359, 2007.

[13] P. M. Meaney, M. W. Fanning, T. Raynolds, et al., "Initial clinical experience with microwave breast imaging in women with normal mammography," Academic Radiology, vol. 14, no. 2, pp. 207-218, 2007.

[14] A. E. Souvorov, A. E. Bulyshev, S. Y. Semenov, R. H. Svenson, and G. P. Tatsis, "Two-dimensional computer analysis of a microwave flat antenna array for breast cancer tomography," IEEE Transactions on Microwave Theory and Techniques, vol. 48, no. 8, pp. 1413-1415, 2000.

[15] H. Jiang, C. Li, D. Pearlstone, and L. L. Fajardo, "Ultrasoundguided microwave imaging of breast cancer: tissue phantom and pilot clinical experiments," Medical Physics, vol. 32, no. 8, pp. 2528-2535, 2005.

[16] A. Massa, D. Franceschini, G. Franceschini, M. Pastorino, M. Raffetto, and M. Donelli, "Parallel GA-based approach for microwave imaging applications," IEEE Transactions on Antennas and Propagation, vol. 53, no. 10, pp. 3118-3127, 2005.

[17] S. Liewei, L. W. Nolte, Q. Z. Zhong, and Q. H. Liu, "Performance analysis for Bayesian microwave imaging in decision aided breast tumor diagnosis," in Proceedings of IEEE International Symposium on Biomedical Imaging (ISBI '02), pp. 10391042, Washington, DC, USA, July 2002.

[18] S. C. Hagness, A. Taflove, and J. E. Bridges, "Two-dimensional FDTD analysis of a pulsed microwave confocal system for breast cancer detection: fixed-focus and antenna-array sensors," IEEE Transactions on Biomedical Engineering, vol. 45, no. 12, pp. 1470-1479, 1998.

[19] X. Yun, E. C. Fear, and R. H. Johnston, "Compact antenna for radar-based breast cancer detection," IEEE Transactions on Antennas and Propagation, vol. 53, no. 8, pp. 2374-2380, 2005.

[20] R. G. Martin, C. M. de Jong van Coevorden, M. F. Pantoja, S. G. Garcia, and A. R. Bretones, "Ultra-broadband antenna design and characterization," in Proceedings of the 2nd International Conference on Electromagnetic Near-Field Characterization \& Imaging (ICONIC '05), pp. 41-46, Barcelona, Spain, June 2005.

[21] A. Karlsson, "Physical limitations ofantennas in a lossy medium," IEEE Transactions on Antennas and Propagation, vol. 52, no. 8, pp. 2027-2033, 2004.
[22] P. Kosmas and C. M. Rappaport, "FDTD-based time reversal for microwave breast cancer detection-localization in three dimensions," IEEE Transactions on Microwave Theory and Techniques, vol. 54, no. 4, pp. 1921-1927, 2006.

[23] T. Takenaka, H. Zhou, and T. Tanaka, "Inverse scattering for a three-dimensional object in the time domain," Journal of the Optical Society of America A, vol. 20, no. 10, pp. 1867-1874, 2003.

[24] A. Abubakar, P. M. van den Berg, and J. J. Mallorqui, "Imaging of biomedical data using a multiplicative regularized contrast source inversion method," IEEE Transactions on Microwave Theory and Techniques, vol. 50, no. 7, pp. 1761-1771, 2002.

[25] A. Fhager, P. Hashemzadeh, and M. Persson, "Reconstruction quality and spectral content of an electromagnetic timedomain inversion algorithm," IEEE Transactions on Biomedical Engineering, vol. 53, no. 8, pp. 1594-1604, 2006.

[26] S. Y. Semenov, A. E. Bulyshev, A. Abubakar, et al., "Microwavetomographic imaging of the high dielectric-contrast objects using different image-reconstruction approaches," IEEE Transactions on Microwave Theory and Techniques, vol. 53, no. 7, pp. 2284-2294, 2005.

[27] D. Li, P. M. Meaney, T. Raynolds, S. A. Pendergrass, M. W. Fanning, and K. D. Paulsen, "Parallel-detection microwave spectroscopy system for breast imaging," Review of Scientific Instruments, vol. 75, no. 7, pp. 2305-2313, 2004.

[28] R. M. Sega, "Infrared detection of microwave induced surface currents on flat plates," Final Technical Report Air Force Academy, 1982.

[29] C. A. Balanis, Antenna Theory Analysis and Design, John Wiley \& Sons, New York, NY, USA, 2nd edition, 1997.

[30] B. Drozd and W. T. Joines, "Comparison of coaxial dipole antennas for applications in the near-field and far-field regions," Microwave Journal, vol. 47, no. 5, pp. 160-176, 2004.

[31] S. P. Poplack, K. D. Paulsen, A. Hartov, et al., "Electromagnetic breast imaging: average tissue property values in women with negative clinical findings," Radiology, vol. 231, no. 2, pp. 571580, 2004.

[32] H. Q. Woodard and D. R. White, "The composition of body tissues," British Journal of Radiology, vol. 59, no. 708, pp. 12091218, 1986.

[33] K. R. Foster and J. L. Schepps, "Dielectric properties of tumor and normal tissues at radio through microwave frequencies," Journal of Microwave Power, vol. 16, no. 2, pp. 107-119, 1981.

[34] B. Brooksby, S. Srinivasan, B. W. Pogue, et al., "Quantifying adipose and fibroglandular breast tissue properties using MRIguided NIR tomography," in Optical Tomography and Spectroscopy of Tissue VI, vol. 5693 of Proceedings of SPIE, pp. 255264, San Jose, Calif, USA, January 2005.

[35] D. B. Kopans, Breast Imaging, Lippincott Williams \& Wilkins, Philadelphia, Pa, USA, 2nd edition, 1997.

[36] P. M. Meaney, T. Raynolds, L. Potwin, and K. D. Paulsen, “3point support mechanical steering system for high intensity focused ultrasound," Physics in Medicine and Biology, vol. 52, no. 11, pp. 3045-3056, 2007.

[37] R. E. Collin, Foundations for Microwave Engineering, McGrawHill, New York, NY, USA, 1966.

[38] A. Taflove and S. C. Hagness, Computational Electrodynamics: The Finite-Difference Time-Domain Method, Atech House, Norwood, Mass, USA, 2nd edition, 2000.

[39] J.-P. Berenger, "A perfectly matched layer for the absorption of electromagnetic waves," Journal of Computational Physics, vol. 114, no. 2, pp. 185-200, 1994. 

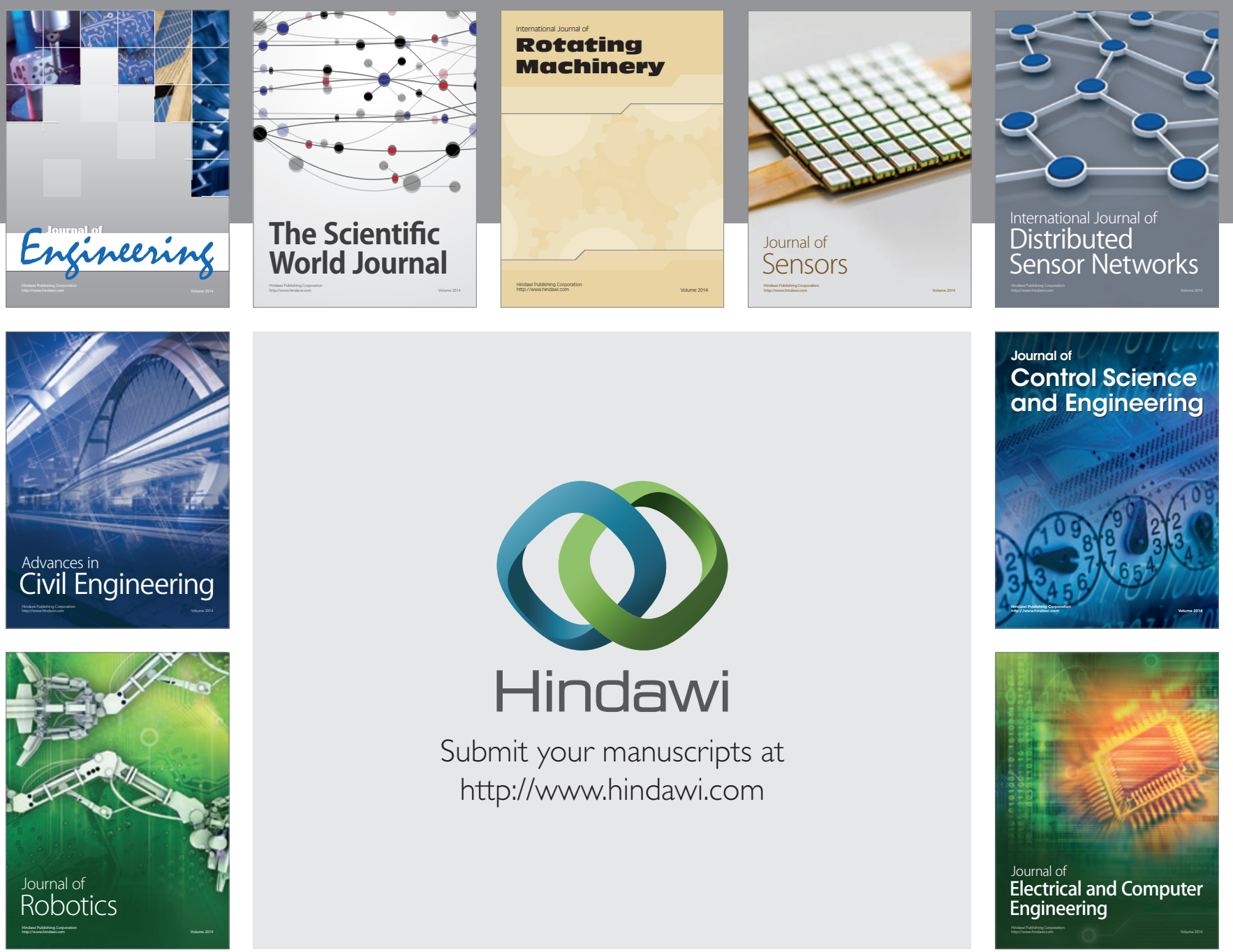

Submit your manuscripts at

http://www.hindawi.com
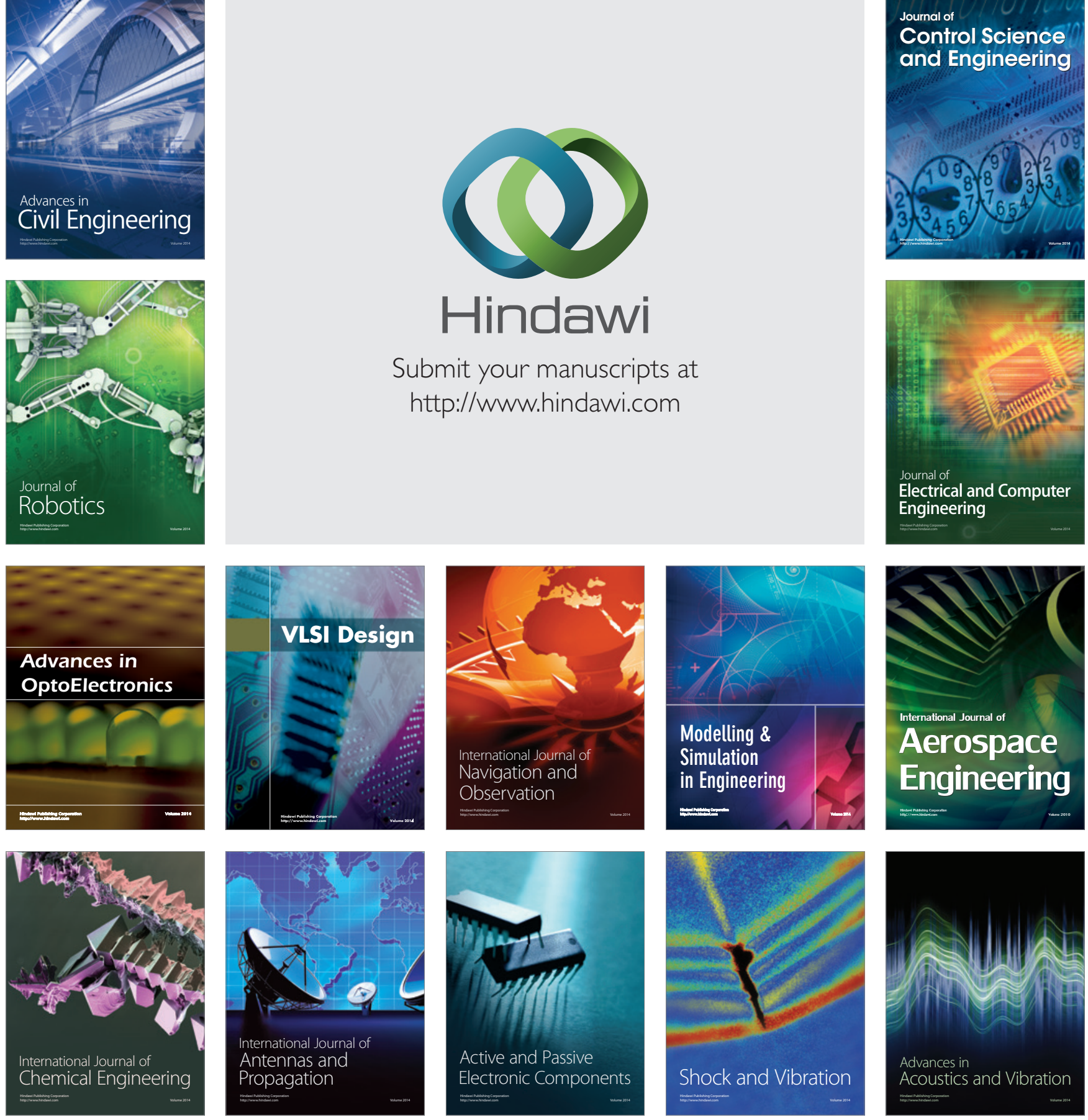